\title{
TRANSPLANTE DE FÍGADO A PARTIR DE DOADORES EM ASSISTOLIA: RESULTADOS INICIAIS DO GRUPO DE TRANSPLANTE DE FÍGADO DO HOSPITAL OSWALDO CRUZ, UNIVERSIDADE DE PERNAMBUCO
}

\author{
LIVER TRANSPLANTATION FROM NON-HEART-BEATING DONORS: INITIAL RESULTS \\ FROM OSWALDO CRUZ UNIVERSITY HOSPITAL'S LIVER TRANSPLANTATION GROUP, \\ PERNAMBUCO UNIVERSITY
}

\author{
Olival Lucena, TCBC-PE' ${ }^{1}$; Américo Gusmão Amorim²; \\ Bernardo Sabat $^{2}$; Luiz Carlos L. Adeodato ${ }^{2}$; Luiz Eduardo Correia Miranda, TCBC-SP'; \\ Cláudio Moura Lacerda, TCBC-PE
}

\begin{abstract}
RESUMO: Objetivo: Relatar a experiência inicial do Grupo de Transplante de Fígado do Hospital Universitário Oswaldo Cruz, Recife - PE, com doadores de fígado em assistolia. Método: Foram revistos os dados referentes a seis transplantes hepáticos, realizados entre outubro de 2002 e setembro de 2004, com órgãos obtidos de doadores em assistolia. Resultados: Não houve disfunção primária do enxerto. Complicações biliares e vasculares não foram observadas. Todos os pacientes receberam alta hospitalar em boas condições clínicas. Conclusões: Embora se trate de uma experiência pequena, bons resultados iniciais foram obtidos com transplante hepáticos realizados a partir de doadores em assitolia (Rev. Col. Bras. Cir. 2005; 32(5): 270-272).
\end{abstract}

Descritores: Transplante de fígado; Doadores de tecidos; Fígado; Parada cardíaca; Seleção de pacientes.

\section{INTRODUÇÃO}

O transplante hepático tornou-se o procedimento de escolha para o tratamento da doença hepática terminal ${ }^{1}$. O sucesso do tratamento resultou no aumento da demanda de órgãos ${ }^{2}$, sem o aumento correspondente de sua oferta. O número de candidatos ao transplante hepático rapidamente excedeu o número de doadores. O tempo de espera por um órgão e os óbitos ocorridos sem que o transplante de fígado tenha sido realizado também aumentaram ${ }^{3}$. Em janeiro de 2004 havia 5.587 pacientes inscritos na lista de espera por um transplante de fígado no Brasil. Foram realizados 955 transplantes e o número de inscritos em janeiro de 2005 aumentou para 6.286 pacientes, dados da Associação Brasileira Transplante de Órgãos (www.abto.org.br).

Para enfrentar esse problema, numerosas estratégias têm sido propostas. O uso de fígados de doadores anteriormente considerados inadequados ${ }^{4}, o$ transplante intervivos e a divisão do fígado com o objetivo de obter dois enxertos viáveis, são as principais delas ${ }^{3}$. O uso de fígados obtidos de doadores em assistolia é um recurso que tem se mostrado viável para aumentar a oferta de órgãos para transplante ${ }^{5,6}$, representando até $5 \%$ da oferta de fígados em grandes centros $^{7}$. O objetivo desse trabalho é relatar a experiência inicial do Grupo de Transplante de Fígado do Hospital Universitário Oswaldo Cruz, Universidade de Pernambuco, com transplantes de fígados realizados a partir de doadores em assistolia.

\section{MÉTODO}

Foram analisados os prontuários médicos de pacientes submetidos a transplante hepático a partir de doadores em assistolia, todos do tipo IV segundo a classificação de Maastrich, entre outubro de 2002 e setembro de 2004, no Hospital Universitário Oswaldo Cruz. Todos os pacientes apresentavam declaração de morte encefálica segundo as exigências da legislação brasileira.

Três critérios foram exigidos para o aproveitamento dos órgãos:

1. avaliação geral do doador quanto à qualidade do fígado, baseada em dados laboratoriais de função hepática, considerada boa ou excelente;

2. aspecto macroscópico normal do fígado, ao inventário da cavidade;

3. mudança do aspecto macroscópico do fígado quanto à temperatura e à cor do órgão, que deve ser homogênea durante a perfusão portal e arterial com solução de preservação.

Foram avaliados ainda dados referentes aos doadores, ao procedimento cirúrgico, e à evolução pós-operatória.

\section{RESULTADOS}

Foram operados seis pacientes, quatro homens e duas mulheres, com idade entre quatro e 53 anos (média $=28$ anos).

1. Cirurgião do Serviço de Cirurgia Geral e Transplante de Fígado do HUOC - UPE.

2. Professor adjunto de Cirurgia e Cirurgião do Serviço de Cirurgia Geral e Transplante de Fígado do HUOC - UPE

3. Prof. Titular de Cirurgia Abdominal e Chefe do Serviço de Cirurgia Geral e Transplante de Fígado do HUOC - UPE.

Recebido em: 02/05/2005

Aceito para publicação em: 09/09/2005

Conflito de interesse: nenhum

Fonte de financiamento: nenhuma

Trabalho realizado no Serviço de Cirurgia Geral e Transplante de Fígado do Hospital Universitário Oswaldo Cruz da Universidade de Pernambuco. 
A causa da morte foi traumatismo craniano em quatro, e acidente vascular cerebral em dois. Todos os doadores apresentaram a parada cardíaca na presença da equipe de captação de órgãos.

O tempo decorrido entre a parada cardíaca e o início da perfusão hepática com solução de preservação foi de oito a 30 minutos. $\mathrm{O}$ tempo de isquemia fria variou entre cinco horas e 50 minutos a 11 horas e 22 minutos. O tempo de isquemia quente variou entre 50 minutos e 82 minutos. Não houve complicações maiores no período intra-operatório em nenhuma das cirurgias relatadas.

Os pacientes permaneceram durante um a sete dias na UTI. O período de permanência hospitalar variou entre oito e 26 dias. Os valores de transaminases atingiram cifras até 10 vezes maiores que o limite normal. O tempo de protrombina mostrou-se prolongado. Um paciente com os piores valores laboratoriais evoluiu com rápida melhora clínica e bioquímica. Trombocitopenia foi observada em quatro casos. Todos os pacientes receberam alta hospitalar em boas condições clínicas. Houve dois óbitos tardios, não associados à característica do doador. $\mathrm{O}$ primeiro óbito, ocorreu no $56^{\circ}$ dia pós-operatório após biópsia hepática e hemotórax maciço. O segundo óbito, ocorreu no $4^{\circ}$ mês pós-operatório por provável doença enxerto-versus-hospedeiro. Os outros pacientes estão vivos e com boa qualidade de vida. Não foram observadas complicações biliares ou vasculares.

\section{DISCUSSÃO}

O primeiro transplante hepático do Nordeste do Brasil foi realizado em $1993^{\circ}$. A partir de agosto de 1999, o grupo ligado ao Hospital Universitário Oswaldo Cruz iniciou um programa de transplante hepático capaz de atender a parte da demanda regional. Até dezembro de 2004, 95 pacientes foram transplantados, 34 deles no último ano (dados não publicados). Apesar das estratégias atuais para aumentar o número de cirurgias, há grande número de óbitos entre pacientes que aguardam pelo enxerto, uma vez que o número de doadores de órgãos para transplante é insuficiente para atender a demanda existente. $\mathrm{O}$ transplante de fígado a partir de doadores em assistolia representa uma maneira de aumentar a oferta de órgãos ${ }^{5}$. Preocupações existem com relação ao aumento do tempo de isquemia a quente, causado pelo tempo decorrido entre a parada cardíaca e o início da perfusão hepática com soluções de preservação. O epitélio biliar é altamente sensível à lesão por reperfusão e, portanto complicações biliares podem ocorrer nos pacientes receptores de desse tipo de enxer- to ${ }^{9}$. A disfunção primária do órgão, resultante da lesão por reperfusão pode ser agravada pelo mesmo motivo ${ }^{10,11}$. Não obstante essas preocupações sejam bem fundamentadas, grupos importantes publicaram dados que demonstram que o transplante de fígado a partir de doadores em assistolia apresenta resultados similares, em longo prazo, àqueles realizados com doadores portadores de morte cerebral e sob técnicas usuais ${ }^{6}$. O grupo da Universidade de Pittsburgh publicou estudo com 10 receptores de doadores em assitolia com um ano de sobrevida para o paciente e para o enxerto de $95 \%$ e $86 \%$ respectivamente ${ }^{12}$. Na série da Universidade da Califórnia, Los Angeles, para 13 paciente transplantados em condições semelhantes às do presente trabalho, a sobrevida para o paciente e para o enxerto em um ano, foram de $85 \%$ e $77 \%$ respectivamente ${ }^{13}$. Os melhores resultados foram obtidos com tempo de assitolia inferior a 30 minutos ${ }^{13,14}$.

Embora a possibilidade do uso de órgãos de doadores em assistolia possa aumentar o número de órgãos disponíveis para transplante hepático, alternativas para esse fim ainda não foram esgotadas em nosso meio. O número de doações por ano por milhão de habitantes no Brasil é muito inferior ao de países que possuem sistema de captação de órgãos realmente competentes. Investimentos para a capacitação e profissionalização das equipes das Centrais de Transplante de Órgãos, a adoção de técnicas de procura ativa de doadores, abordagem de familiares por profissionais preparados e campanhas contínuas para educação da população leiga, representam estratégias para aumento do número de doadores que devem ser exploradas pelas autoridades responsáveis pelo planejamento e administração da Centrais de Transplante de Órgãos. As técnicas de divisão do enxerto, e de transplante intervivos ainda são pouco usadas por nossos centros de transplante. Técnicas para o desenvolvimento de meios que permitam a proteção do órgão a ser transplantado, aumentando a chance de sucesso do enxerto, constituem empolgante e atrativo campo de pesquisa científica, ainda pouco explorado. Mesmo em países onde essas técnicas são usadas eficientemente há escassez de órgãos, o que torna necessário o desenvolvimento de novas alternativas para aumentar o número de transplantes.

Em resumo, apesar das preocupações justificáveis com relação aos fígados obtidos de pacientes em assistolia, a experiência preliminar do Grupo de Transplante de Fígado do Hospital Oswaldo Cruz é concordante com os relatos da literatura que sugerem que enxertos obtidos por esse meio aumentam, de forma segura, a oferta de órgãos para transplante hepático.

\footnotetext{
ABSTRACT

Background: The demand for liver transplantation has increasingly exceeded the supply of cadaver donor organs. Hepatic allografts from non-heart-beating donors have been cited as a means to expand the supply of donor livers. Methods: We reviewed the medical records of six patients who had undergone orthotopic liver transplantation from non-heart-beating donors at Oswaldo Cruz University Hospital, Pernambuco University, Brazil, between October 2002 and September 2004. Results: Primary graft non-function, vascular or biliary complications were not observed. Re-transplantation was not required. All patients were discharged from hospital in good clinical conditions. Conclusion: Although more experience is required, good initial results have been obtained with non-heart-beating livers donors by the Oswaldo Cruz University Hospital Group.
}

Key words: Liver transplantation; Tissue donors; Liver; Heart arrest; Patients selection. 


\section{REFERÊNCIAS}

1. Starzl TE, Marchioro TL, VonKaulla KN, et al. Homotransplantation of the liver in humans. Surg Gynecol Obstet. 1963; 117:659-76.

2. Starzl TE, Iwatsuki S, Shaw BW Jr, et al. Orthotopic liver transplantation in 1984. Transplant Proc, 1985, 17:250.

3. Sherlock S, Dooley J, editors. Diseases of the liver and biliary system. 10 ${ }^{\text {th }}$ ed. Oxford: Blackwell; 1997.

4. Cecka JM. Donor and preservation factors. Clin Transpl. 1988;399-408.

5. Fung JJ. Use of non-heart-beating donors. Transplant Proc. 2000; 32(7):1510-1.

6. Manzarbeitia CY, Ortiz JA, Jeon H, et al. Long-term outcome of controlled, non-heart-beating donor liver transplantation. Transplantation. 2004;78(2):211-5.

7. González-Segura C. Donantes de órganos e corazón parado. In: López-Navidad A, Kulisevsky J, Caballero F. El donante de órganos y tejidos: evaluación y manejo. Barcelona: SpringerVerlag; 1997. p.497-507.

8. Lacerda CM, Maia M, Sette M. Carcinoma fibrolamelar recidivado. O primeiro caso de transplante de fígado no Recife. An Fac Med Univ Fed Pernamb.1994;39(2):125-8.
9. Abt P, Crawford M, Desai N, et al. Liver transplantation from controlled non-heart-beating donors: an increase incidence of biliary complications. Transplantation. 2003;75(10):1659-63.

10. Miranda LE, Viaro F, Ceneviva R, et al. Bases experimentais da lesão por isquemia e reperfusão do fígado: revisão. Acta Cir Bras. 2004; 19(1):3-12.

11. Otero A, Gomez-Gutierrez M, Suarez F, et al. Liver transplantation from maastricht category 2 non-heart-beating donors: a source to increase the donor pool? Transplant Proc. 2004; 36(3):747-50.

12. Casavilla A, Ramirez C, Shapiro R, et al. Experience with liver and kidney allografts from non-heart- beating donors. Transplantation. 1995; 59(2):197-203.

13. Busuttil RW, Tanaka K. The utility of marginal donors in liver transplantation. Liver Transpl. 2003; 9(7):651-63.

14. Evanisko MJ, Beasley CL, Brigham LE, et al. Readiness of critical care physicians and nurses to handle requests for organ donation. Am J Crit Care. 1998; 7(1):4-12.

Endereço para correspondência:

Cláudio Moura Lacerda,

Rua Prof. Anunciada Rocha Melo, 87, apto. 501, Madalena

Recife - PE - CEP 50710-390

E-mail: cmlacerda1@hotmail.com

Telefone: (0xx81) 9971-5515 